\title{
SIMULTANEOUS SYSTEMS OF REPRESENTATIVES FOR FINITE FAMILIES OF FINITE SETS
}

\author{
XING-DE JIA
}

(Communicated by Larry J. Goldstein)

\begin{abstract}
Let $h \geq 2$ and $k \geq 1$. It is proved that if $\mathscr{S}=\left\{S_{i}\right\}_{i=1}^{s}$ and $\mathscr{T}=\left\{T_{j}\right\}_{j=1}^{t}$ are two families of nonempty, pairwise disjoint sets such that $\left|S_{i}\right| \leq h,\left|T_{j}\right| \leq k$ and $S_{i} \nsubseteq T_{j}$ for all $i$ and $j$, then the number $N(\mathscr{S}, \mathscr{T})$ of the sets $X$ such that $X$ is a minimal system of representatives for $\mathscr{S}$ and $X$ is simultaneously a system of representatives for $\mathscr{T}$ that satisfies $N(\mathscr{S}, \mathscr{T}) \leq$ $h^{s}\left(1-(h-r) / h^{q+1}\right)^{t}$, where $k=q(h-1)+r$ with $0 \leq r \leq h-2$. This was conjectured by M. B. Nathanson [3] in 1985.
\end{abstract}

1. Introduction. Let $\mathscr{S}=\left\{S_{i}\right\}$ be a family of nonempty sets. The set $X$ is a system of representatives for $\mathscr{S}$ if $X \cap S_{i} \neq \varnothing$ for every $S_{i}$ in $\mathscr{S}$. If $X$ is a system of representatives for $\mathscr{S}$, but no proper subset of $X$ is a system of representatives for $\mathscr{S}$, then $X$ is called a minimal system of representatives for $\mathscr{S}$.

Let $\mathscr{S}=\left\{S_{i}\right\}$ and $\mathscr{T}=\left\{T_{j}\right\}$ be two families of nonempty sets. Let $N(\mathscr{S}, \mathscr{T})$ denote the number of sets $X$ such that $X$ is a minimal system of representatives for $\mathscr{S}$ and $X$ is also a system of representatives for $\mathscr{T}$.

The study of the number $N(\mathscr{S}, \mathscr{T})$ could be usefully applied to investigate asymptotic bases in additive number theory. In 1985, Nathanson [3] made two conjectures on this number, which can be stated as follows.

CONJECTURE 1. Let $h \geq 2$ and $k \geq 1$. There exists a real number $\lambda=\lambda(h, k) \in$ $(0,1)$ with the following property: Let $\mathscr{S}=\left\{S_{i}\right\}_{i=1}^{s}$ be a family of $s$ nonempty, pairwise disjoint sets $S_{i}$ with $\left|S_{i}\right| \leq h$ for all $i$. Let $\mathscr{T}=\left\{T_{j}\right\}_{j=1}^{t}$ be a family of $t$ nonempty, pairwise disjoint sets $T_{j}$ with $\left|T_{j}\right| \leq k$ for all $j$. Suppose $S_{i} \nsubseteq T_{j}$ for all $i$ and $j$. Then

$$
N(\mathscr{S}, \mathscr{T}) \leq h^{s} \lambda^{t} .
$$

CONJECTURE 2. Let $h \geq 2$ and $k \geq 1$. Let $k=q(h-1)+r$, where $q=[k /(h-1)]$ and $0 \leq r \leq h-2$. Define

$$
\lambda^{*}(h, k)=1-(h-r) / h^{q+1} .
$$

Then $\lambda^{*}(h, k)$ is the smallest value of $\lambda$ for which inequality (1) is true for all families $\mathscr{S}$ and $\mathscr{T}$ that satisfy the conditions of Conjecture 1.

It has been proved that these two conjectures are true in many special cases. Early in 1979, Erdös and Nathanson [1] proved that the conjectures hold if $h=$ $k=2$ when they investigated asymptotic additive bases of order 2 in additive number theory. Jia [2] proved in 1986 that the conjectures are true in the case that

Received by the editors October 1, 1986 and, in revised form, March 30, 1987.

1980 Mathematics Subject Classification (1985 Revision). Primary 11B13, 05A05. 
$h=k \geq 2$. In his 1985 paper [3], Nathanson proved the conjectures in some special cases. And he proved that if $\lambda \in(0,1)$ satisfies $N(\mathscr{S}, \mathscr{T}) \leq h^{s} \lambda^{t}$ for all $\mathscr{S}$ and $\mathscr{T}$ that satisfy the conditions of Conjecture 1 , then $\lambda \geq \lambda^{*}(h, k)$. In the present paper, we prove that Conjectures 1 and 2 are true for any $h \geq 2$ and $k \geq 1$.

2. Main result and a lemma. The main result of this paper is

THEOREM. Let $h \geq 2$ and $k \geq 1$. Let $k=q(h-1)+r$, where $q=[k /(h-1)]$ and $0 \leq r \leq h-2$. Then

$$
N(\mathscr{S}, \mathscr{T}) \leq h^{s}\left(1-(h-r) / h^{q+1}\right)^{t}
$$

holds for any finite families $\mathscr{S}$ and $\mathscr{T}$ that satisfy the conditions of Conjecture 1.

In particular, we have

$$
N(\mathscr{S}, \mathscr{T}) \leq h^{s}\left(\left(h^{2}-h+1\right) / h^{2}\right)^{t}
$$

if $h=k \geq 2$, which is a result by Jia [2], and

$$
N(\mathscr{S}, \mathscr{T}) \leq h^{s}(k / h)^{t}
$$

if $h>k \geq 1$.

The following lemma will be used in the proof of the theorem.

LEMMA. Let $h \geq 2$ and $m \geq 1$ with $m \leq L<m h$. If

$$
L-m=u(h-1)-r,
$$

where $u$ is an integer and $0 \leq r \leq h-2$, then

$$
x_{1} \cdots x_{m} \geq h^{u-1}(h-r)
$$

holds for any integers $1 \leq x_{i} \leq h(i=1,2, \ldots, m)$ with $\sum_{i=1}^{m} x_{i}=L$.

PROOF. Let $f\left(x_{1}, \ldots, x_{m}\right)=x_{1} \cdots x_{m}$. It is well known that $f$ has no minimal point inside the inner $\mathscr{D}$ of the domain $\overline{\mathscr{D}}: 1 \leq x_{i} \leq h(i=1, \ldots, m)$ with the restriction $x_{1}+\cdots+x_{m}=L$. Hence the minimal point of $f$ must be on the boundary $\partial \overline{\mathscr{D}}$ of $\overline{\mathscr{D}}$.

Since $L<m h$, it follows from the definition of $u$ that $u \leq m$. First we assume $u=m$, then $L=m h-r$. We prove

$$
f\left(x_{1}, \ldots, x_{m}\right) \geq h^{m-1}(h-r)
$$

by induction on $m$. It is clear that (5) is true if $m=1$. Now assume that (5) holds for any $m^{\prime}<m$. Let $\left(x_{1}, \ldots, x_{m}\right)$ be a minimal point of $f$ on $\partial \overline{\mathscr{D}}$, where $x_{1} \leq x_{2} \leq \cdots \leq x_{m}$. Since $x_{1}=L-\left(x_{2}+\cdots+x_{m}\right) \geq L-(m-1) h=h-r \geq 2$, it follows from $\left(x_{1}, \ldots, x_{m}\right) \in \partial \overline{\mathscr{D}}$ that $x_{m}=h$. Therefore $x_{1}+\cdots+x_{m-1}=L-r=$ $(m-1) h-r$, thus

$$
\begin{aligned}
f\left(x_{1}, \ldots, x_{m}\right) & =x_{1} \cdots x_{m}=h x_{1} \cdots x_{m-1} \\
& \geq h\left(h^{m-2}(h-r)\right)=h^{m-1}(h-r),
\end{aligned}
$$

which proves (5). 
Now assume $u<m$. If $\left(x_{1}, \ldots, x_{m}\right) \in \partial \overline{\mathscr{D}}$ is such that $x_{1} \leq \cdots \leq x_{m}$ and $f\left(x_{1}, \ldots, x_{m}\right)$ is minimal, then $x_{1}=1$. Otherwise, we suppose $2 \leq x_{1} \leq \cdots \leq x_{v}<$ $x_{v+1}=\cdots=x_{m}=h$. Then $\left(x_{1}-1, x_{2}, \ldots, x_{v-1}, x_{v}+1, x_{v+1}, \ldots, x_{m}\right) \in \partial \overline{\mathscr{D}}$, and

$$
\begin{gathered}
f\left(x_{1}-1, x_{2}, \ldots, x_{v-1}, x_{v}+1, x_{v+1}, \ldots, x_{m}\right)=\left(x_{1}-1\right) x_{2} \cdots x_{v-1}\left(x_{v}+1\right) h^{m-v} \\
=x_{1} x_{2} \cdots x_{v} h^{m-v}-x_{2} \cdots x_{v-1} h^{m-v}\left(x_{v}+1-x_{1}\right)<f\left(x_{1}, x_{2}, \ldots, x_{m}\right),
\end{gathered}
$$

which contradicts the minimality of $f\left(x_{1}, \ldots, x_{m}\right)$. Therefore $x_{2}+\cdots+x_{m}=$ $L-1=u(h-1)-r+(m-1)$, thus $x_{1} \cdots x_{m}=x_{2} \cdots h^{u-1}(h-r)$. This shows that we can assume that $u=m$. Hence the proof of the lemma is complete.

3. The proof of the theorem. Let $\mathscr{S}=\left\{S_{i}\right\}_{i=1}^{s}$ and $\mathscr{T}=\left\{T_{j}\right\}_{j=1}^{t}$ be two finite families of finite sets that satisfy the conditions of Conjecture 1. Let $S_{s+1}$ be a set of $h$ elements such that $S_{s+1}$ does not intersect any $S_{i}$ in $\mathscr{S}$. Taking $\mathscr{S}^{\prime}=\mathscr{S} \cup\left\{S_{s+1}\right\}$, we have $N\left(\mathscr{S}^{\prime}, \mathscr{T}\right) \geq h N(\mathscr{S}, \mathscr{T})$. This allows us to assume that the integer $s$ is sufficiently large. Therefore we may assume without loss of generality that

$$
\begin{array}{ll}
\left|S_{i}\right|=h & \text { for } i=1,2, \ldots, s \\
\left|T_{j}\right|=k & \text { for } j=1,2, \ldots, t
\end{array}
$$

and

$$
S=\bigcup_{i=1}^{s} S_{i} \supseteq T=\bigcup_{j=1}^{t} T_{j} .
$$

We will prove the theorem by induction on $t$ for fixed $s>2 k t$. If $t=0$ then $N(\mathscr{S}, \mathscr{T})=h^{s}$. Let $t \geq 1$ and assume that (2) holds for any $0 \leq t^{\prime}<t$ and any $s$.

We consider $T_{t}$. Let $\left\{S_{1}, \ldots, S_{m}\right\}$ be the set of those $S_{i}$ that intersect $T_{t}$. Denote

$$
\left|S_{i} \cap T_{t}\right|=n_{i} \quad \text { for } i=1, \ldots, m,
$$

then $n_{1}+\cdots+n_{m}=k$, and $S_{i} \nsubseteq T_{t}$ implies that $1 \leq n_{i} \leq h-1$ for $i=$ $1, \ldots, m$. Let $S_{i}=\left\{a_{i 1}, a_{i 2}, \ldots, a_{i h}\right\}$, where $a_{i 1}, \ldots, a_{i n_{i}} \in T_{t}$ for $i=1, \ldots, m$. Since $s>2 k t$, there exist $m S_{i}$ in $\mathscr{S}$, say $S_{m+1}, \ldots, S_{2 m}$, such that $S_{i} \cap T=\varnothing$ for $i=m+1, \ldots, 2 m$. Let $S_{i}=\left\{a_{i 1}, a_{i 2}, \ldots, a_{i h}\right\}$ for $i=m+1, \ldots, 2 m$.

We construct

$$
\begin{aligned}
& S_{i}^{\prime}=\left\{a_{i 1}, \ldots, a_{i n_{i}}, a_{m+i, n_{i}+1}, \ldots, a_{m+i, h}\right\} \\
& S_{m+i}^{\prime}=\left\{a_{m+i, 1}, \ldots, a_{m+i, n_{i}}, a_{i, n_{i}+1}, \ldots, a_{i h}\right\}
\end{aligned}
$$

for $i=1, \ldots, m$. Let

$$
\mathscr{S}^{\prime}=\left(\mathscr{S} \backslash\left\{S_{1}, \ldots, S_{2 m}\right\}\right) \cup\left\{S_{1}^{\prime}, \ldots, S_{2 m}^{\prime}\right\} .
$$

Then $\mathscr{S}^{\prime}$ and $\mathscr{T}$ satisfy the conditions of Conjecture 1, and the corresponding integers $s$ and $t$ do not change.

Let $X$ be a simultaneous system of representatives counted in $N(\mathscr{S}, \mathscr{T})$. Denote

$$
\begin{aligned}
X \cap S_{i} & =\left\{x_{i}\right\} \quad \text { for } i=1, \ldots, m ; \\
X_{1} & =X \backslash \bigcup_{i=1}^{2 m} S_{i} .
\end{aligned}
$$


Then it follows from $S_{i} \cap T=\varnothing$ for $i=m+1, \ldots, 2 m$ that exactly $h^{m}$ simultaneous systems $X$ of representatives counted in $N(\mathscr{S}, \mathscr{T})$ contain $\left\{x_{1}, \ldots, x_{m}\right\} \cup X_{1}$.

Suppose $x_{j} \in S_{i_{j}}^{\prime}$ for $j=1, \ldots, m$. Then $i_{j}=j$ or $j+m$, and $S_{i_{j}+m}^{\prime} \cap$ $\left(\left\{x_{1}, \ldots, x_{m}\right\} \cup X_{1}\right)=\varnothing$ for $j=1, \ldots, m$, where the subscripts of $S_{i}^{\prime}$ 's are regarded as elements of the group $\mathbf{Z} /(2 m)$. Therefore for any $x_{j+m} \in S_{i_{j}+m}^{\prime}$ for $j=1, \ldots, m$, taking $X^{\prime}=\left\{x_{1}, \ldots, x_{2 m}\right\} \cup X_{1}$, we see that $X^{\prime}$ is a minimal system of representatives for $\mathscr{S}^{\prime}$ that contains a system of representatives for $\mathscr{T}$, and $X^{\prime}$ contains $\left\{x_{1}, \ldots, x_{m}\right\} \cup X_{1}$. Since there are $h^{m}$ different simultaneous systems $X$ of representatives counted in $N\left(\mathscr{S}^{\prime}, \mathscr{T}\right)$. Therefore $N\left(\mathscr{S}^{\prime}, \mathscr{T}\right) \leq N(\mathscr{S}, \mathscr{T})$. Hence we may assume that $S_{i} \cap T_{t}=S_{i} \cap T$ for $i=1,2, \ldots, m$.

Let $\mathscr{S}^{\prime}=\left\{S_{i}\right\}_{i=m+1}^{s}$ and $\mathscr{T}^{\prime}=\left\{T_{j}\right\}_{j=1}^{t-1}$. Clearly $\mathscr{S}^{\prime}$ and $\mathscr{T}^{\prime}$ satisfy the conditions of Conjecture 1 (for $s-m$ and $t-1$ ). For any $X_{1}$ counted in $N\left(\mathscr{S}^{\prime}, \mathscr{T}^{\prime}\right)$, there are $h^{m}-\left(h-n_{1}\right) \cdots\left(h-n_{m}\right)$ different $X$ counted in $N(\mathscr{S}, \mathscr{T})$ containing $X_{1}$. Since

$$
L=\sum_{i=1}^{m}\left(h-n_{1}\right)=m h-\sum_{i=1}^{m} n_{i}=m h-k,
$$

then $L-m=m(h-1)-k=m(h-1)-q(h-1)-r=(m-q)(h-1)-r$. Hence by the lemma, we have $\left(h-n_{1}\right) \cdots\left(h-n_{m}\right) \geq h^{m-q-1}(h-r)$. Therefore

$$
\begin{aligned}
N(\mathscr{S}, \mathscr{T}) & \leq\left(h^{m}-\left(h-n_{1}\right) \cdots\left(h-n_{m}\right)\right) N\left(\mathscr{S}^{\prime}, \mathscr{T}^{\prime}\right) \\
& \leq\left(h^{m}-h^{m-q-1}(h-r)\right) h^{s-m}\left(1-(h-r) / h^{q+1}\right)^{t-1} \\
& =h^{s}\left(1-(h-r) / h^{q+1}\right)^{t} .
\end{aligned}
$$

This completes the proof of the theorem.

Nathanson [3] has given an example of two families $\mathscr{S}$ and $\mathscr{T}$ of finite sets that satisfy the conditions of Conjecture 1 , for which,

$$
N(\mathscr{S}, \mathscr{T})=h^{s}\left(1-(h-r) / h^{q+1}\right)^{t} .
$$

Therefore the upper bound of $N(\mathscr{S}, \mathscr{T})$ in the theorem above is the best possible result.

ACKNOWLEDGement. I would like to express my thanks to Professor M. B. Nathanson for his helpful suggestion, which led to the work of this paper, and to the referee for several corrections.

\section{REFERENCES}

1. P. Erdös and M. B. Nathanson, Systems of distinct representatives and minimal bases in additive number theory, Number Theory, Carbondale 1979 (M. B. Nathanson, ed.), Lecture Notes in Math., vol. 751, Springer-Verlag, Berlin and New York, 1979, pp. 89-107.

2. Jia Xing-De, On an open combinatorial problem of Erdös and Nathanson, Chinese Ann. Math. (to appear).

3. M. B. Nathanson, Simultaneous systems of representatives for families of finite sets, Proc. Amer. Math. Soc. (to appear). CHINA

Department of Mathematics, Qufu Normal University, Qufu, Shandong,

Department of Mathematics, Graduate School and University Center, City University of NeW York, New YORK, New York 10036 (Current address) 